\section{Vigencia del efecto placebo: su biología desde la genética a la conducta}

\author{
DIANA ACEITUNO V. ${ }^{1, a}$, JAIME SANTANDER ${ }^{2}$
}

\section{Biological and genetic mechanisms underlying the placebo effect}

The placebo effect has been seldom studied in the history of medicine. However, during the last decades, the great impact of this phenomenon in clinical practice, ranging from surgical to psychiatric field, has been revealed. Research elucidated both the psychological mechanisms and genetic polymorphisms that affect the susceptibility of individuals to express this phenomenon. We herein review the psychological mechanisms, brain structures (anterior cingulate cortex, nucleus accumbens, dorsolateral prefrontal cortex, insular cortex, thalamus) and neurotransmission systems involved (opioid, dopaminergic, cannabinoid, serotoninergic, cholecystokinin). These are the clue to recognize the polymorphisms that have been identified so far. The biological basis of both the placebo effect and its alter ego, the nocebo effect, are well recognized, and related to corresponding psychological processes. Finally, the implications of the findings in clinical practice and medical training are discussed.

(Rev Med Chile 2017; 145: 775-782)

Key words: Genetics; Neurobiology; Nocebo Effect; Placebo Effect.

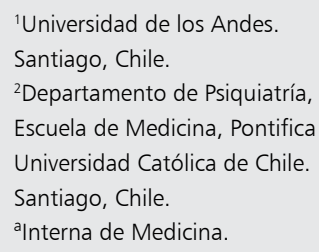

$\mathrm{E}$ n la práctica clínica y la investigación médica observamos que la administración de agentes farmacológicos se acompaña tanto de efectos beneficiosos como perjudiciales que no son explicados por la farmacodinamia del medicamento. A estos efectos beneficiosos percibidos por el paciente y el personal médico se les conoce como efecto placebo (EP) y a aquellos efectos perjudiciales se les denomina efecto nocebo (EN). El EP es resultado de una interacción cuerpo-mente compleja, evocada en una dinámica en la cual la oferta de tratar una condición disfórica o mórbida preexistente con una intervención inerte o inefectiva, resulta en la restauración del bienestar del individuo ${ }^{1}$. Por otro lado, el EN se conceptualiza como una respuesta disfórica o mórbida al tratar a un individuo con una sustancia inactiva o placebo, presentando éste vivencias desagradables tales como dolor de cabeza, astenia o mareos, pudiendo alcanzar prevalencias cercanas a $20 \%{ }^{2}$.
Si bien resulta difícil comparar el EP en distintas enfermedades debido a los diversos factores que intervienen en los estudios correspondientes ${ }^{3}$, revisiones sistemáticas muestran que el beneficio de los medicamentos atribuido al EP llega a $62 \%$ en dolor neuropático y $40 \%$ en fibromialgia y síndrome de piernas inquietas ${ }^{4}$. En el ámbito psiquiátrico, el EP es menor en desórdenes psicóticos que en desórdenes neuróticos, reportándose una tasa promedio de $25 \%$ en pacientes con esquizofrenia ${ }^{5}$ y $67,6 \%$ en depresión unipolar ${ }^{6}$. La transversalidad del fenómeno alcanza el ámbito quirúrgico, observándose que la extracción de cartílago degenerado con artroscopía de rodilla no es más efectiva para el manejo del dolor crónico al compararla con cirugía placebo y que la cirugía placebo en pacientes con Parkinson es capaz de aliviar algunos síntomas por períodos prolongados de tiempo ${ }^{7}$.

A pesar de la evidente magnitud de este fenómeno, la historia del placebo se caracterizó por 
críticos que cuestionaron si este tenía algún sustrato biológico en sí mismo -constituyéndose en una identidad neurofisiológica propia- o si sólo era producto de la subjetividad misma del paciente, quien comunicaba cambios en su realidad como consecuencia de su interpretación. Otro obstáculo para el reconocimiento de su autonomía es la multiplicidad de variables que pueden resultar confundentes, tales como el efecto de regresión a la media, la evolución natural de la enfermedad, sesgos en los estudios, el efecto Hawthorne, cointervenciones no identificadas o la observación de inconsistencias en la reproducción del EP en un mismo individuo. Respecto a esto último, Kradin postuló que la generación de respuesta placebo no sigue un modelo linear, de modo que leves cambios en condiciones iniciales pueden desencadenar eventos finales totalmente diferentes ${ }^{8}$.

Entre tanto escepticismo, se han realizado múltiples investigaciones en pos de la exploración de aquel sustrato orgánico que explique el procesamiento y dinamismo del EP. El objetivo de este trabajo es realizar una revisión dirigida de la literatura internacional de los últimos diez años y de estudios relevantes previos explorando las bases biológicas del EP/EN.

\section{Estructuras implicadas en el efecto placebo y nocebo}

Se ha demostrado que ciertos circuitos del sistema nervioso central (SNC) -que participan en la percepción y la integración de la experiencia del dolor- responden a la manipulación cognitiva. Los procesos cognitivos y emocionales de orden superior involucrados en la habilitación y modulación del EP pueden entenderse del siguiente modo: 1) Expectativas y creencias: la evaluación cognitiva y la creencia de analgesia gatillan el EP; 2) Alivio de la ansiedad: la administración de placebo genera analgesia a través de la reducción de la ansiedad experimentada por el sujeto; 3) La hipótesis del condicionamiento: a través de experiencias previas el organismo aprehende respuestas; 4) La hipótesis "respuesta apropiada a la sensación": establece que el dolor y la analgesia son experiencias dadas posterior a una evaluación preconsciente de estímulos internos y sensoriales?.

Los estudios en neuroimágenes que investigan la analgesia placebo se enfocan en observar si las áreas implicadas en el procesamiento del dolor varían en su actividad, hallándose que en la construcción cronológica del EP cada estructura adquiere un rol por fases: 1) En el estado basal, la conexión entre corteza prefrontal medial izquierda e ínsula y las oscilaciones de alta frecuencia en la corteza prefrontal dorsolateral (CPFDL) izquierda son predictores de la magnitud de la respuesta placebo; 2) Luego, en el proceso de anticipación, fenómenos como el aumento de actividad en la red frontoparietal, la disminución de la actividad en circuito cortezatemporal-ínsula, la conexión entre la red frontoparietal y corteza prefrontal/ corteza cingulada anterior (CCA) y la conexión entre sistema sensoriomotor $y$ ambos hemisferios cerebelosos funcionan como predictores positivos; 3) Posteriormente, en el procesamiento del dolor, se observa que la disminución de la actividad en regiones límbicas y paralímbicas colaboran en la formación de este fenómeno; 4) Finalmente, en la condición misma de analgesia placebo existe reducción de la actividad en ínsula, CCA, tálamo, amígdala y CPFDL derecha ${ }^{10}$.

La importancia de la CPFDL durante la anticipación del alivio del dolor se ha presentado como un elemento clave, teniendo la actividad de ésta una correlación positiva con la magnitud del efecto placebo ${ }^{11}$. El alterar la función de esta estructura-encargada de procesos cognitivos como regulación de las emociones- genera reducción del $\mathrm{EP}^{12}$. Concordante con lo anterior, el grado de pérdida de la función ejecutiva en pacientes con Alzheimer se correlaciona negativamente con la fuerza del efecto placebo ${ }^{13}$. La suma de evidencia involucra a la CPFDL en la mantención y actualización de las expectativas que inducen y conducen el EP, ejerciendo también control activo en la percepción del dolor al modular vías córtico-subcorticales y córtico-corticales ${ }^{14}$. Por otro lado, al intentar caracterizar el EN, se ha descrito la activación de regiones cerebrales implicadas en el procesamiento cognitivo-emocional del dolor tales como CCA, ínsula, opérculo, corteza orbitofrontal y corteza prefrontal lateral, observándose, además, que la activación del hipocampo se asocia al aumento de la ansiedad anticipatoria durante el procesamiento del estímulo doloroso ${ }^{15}$. La relación entre las estructuras del SNC implicadas en la analgesia placebo y las etapas del procesamiento cognitivo se resumen a continuación en la Figura 1. 


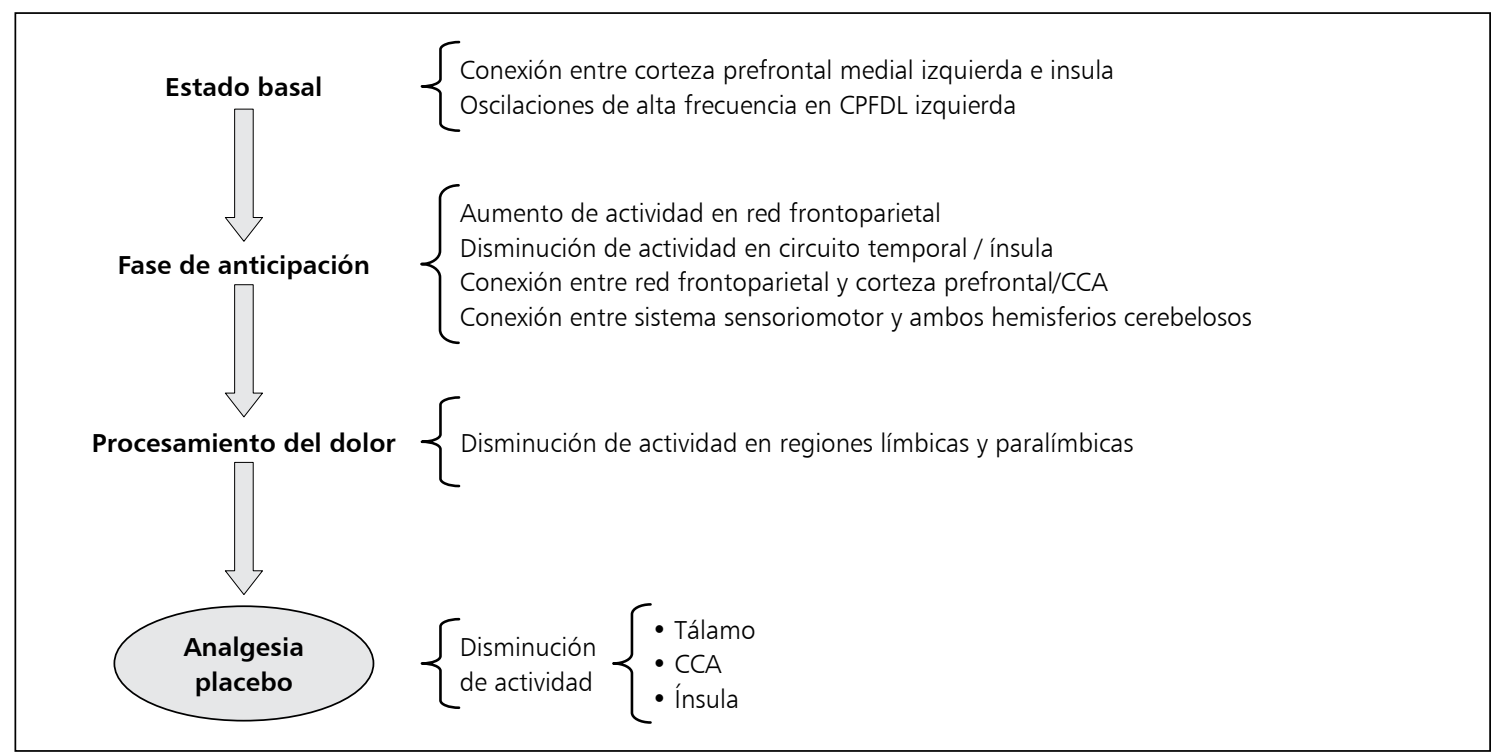

Figura 1. Resumen de las estructuras involucradas en el efecto placebo. CPFDL: corteza prefrontal dorsolateral. CCA: corteza cingulada anterior.

\section{Neurotransmisores implicados en el efecto placebo}

Distintos sistemas endógenos participan en el EP/EN. Los principales hallazgos se resumen en la Tabla 1.

\section{Placebo y sistema opioide}

En 1978, Levine et al demostraron que la naloxona reducía la respuesta placebo en el contexto de dolor post-operatorio ${ }^{16}$, constituyendo la primera prueba de que el sistema opioide endógeno (SOE) estaba implicado en la analgesia placebo. Posteriormente, el efecto de la analgesia placebo fue estimado como equivalente a $8 \mathrm{mg}$ de morfina ${ }^{17}$.

Benedetti et al, al analizar la participación del condicionamiento y de la expectativa en el fenómeno de analgesia placebo, mostró que si bien factores cognitivos están asociados a la activación del SOE, el condicionamiento previo es capaz de activar otros mecanismos para la analgesia dependiendo del agente implicado en el condicionamiento (el condicionamiento previo con ketorolaco -sin asociación a sugestión sobre expectación de analgesia- no otorga una analgesia reversible a la naloxona $)^{18}$. La participación del
SOE fue corroborado posteriormente al hallar mayor cantidad de endorfinas en el líquido cefalorraquídeo de respondedores placebo versus no respondedores ${ }^{19}$.

En 2002, un estudio utilizando tomografía por emisión de positrones $(\mathrm{PET})^{20}$ reveló patrones de activación cerebral en el flujo sanguíneo de la CCA que coincidían tanto en condiciones de analgesia placebo como en condiciones de administración de remifentanilo (agonista del receptor opioide- $\mu$ $[\mathrm{RO}-\mu]$ ), sugiriendo, además, que las diferencias interindividuos en el EP pueden deberse a una diferente concentración o función de $\mathrm{RO}-\mu$. En 2005 , surge la primera evidencia de que la administración de placebo con propiedades analgésicas implícitas está asociada a la activación del SOE $\mathrm{y}$ de $\mathrm{RO}-\mu$ situados en distintas regiones cerebrales tales como CCA, CPFDL, corteza insular anterior (CIA) y núcleo accumbens (NA). Estas activaciones regionales fueron correlacionadas con menores grados de intensidad del dolor (CCA, CIA, NA), menor desagrado por el dolor (CCA), menor puntaje sensorial en el cuestionario del dolor McGill (CIA y CCA), menor puntaje afectivo en este mismo cuestionario (NA) y menor puntaje total en el estado emocional negativo de los voluntarios (NA). Así, las regiones implicadas en 
Tabla 1. Neurotransmisores implicados en efecto placebo

\begin{tabular}{|c|c|c|}
\hline Sistema implicado & Hallazgos & Referencia \\
\hline \multirow[t]{5}{*}{ Sistema opioide } & $\begin{array}{l}\text { Naloxona reduce analgesia placebo en dolor post } \\
\text { operatorio }\end{array}$ & Levine et al $(1978)^{16}$ \\
\hline & Expectativa de analgesia placebo activa SOE & Amanzio et al (1999) ${ }^{18}$ \\
\hline & $\begin{array}{l}\text { LCR con mayor cantidad de endorfinas en } \\
\text { respondedores }\end{array}$ & Lipmann et al $(1990)^{19}$ \\
\hline & $\begin{array}{l}\text { RO- } \mu \text { en codiciones de analgesia placebo son activados } \\
\text { en } C C A, N A, C I A, C P F D L\end{array}$ & Zubieta et al $(2005)^{21}$ \\
\hline & $\begin{array}{l}\text { Polimorfismo } \mathrm{A} 118 \mathrm{G} \text { del } \mathrm{RO}-\mu \text { genera variaciones en } \\
\text { disponibilidad de } \mathrm{RO}-\mu \text { y en la activación dopaminérgica } \\
\text { en la experiencia de dolor y analgesia placebo }\end{array}$ & Pecina et al $(2015)^{23}$ \\
\hline \multirow[t]{2}{*}{ Sistema cannabinoide } & $\begin{array}{l}\text { Receptores CB1 y CB2 son participantes en los mecanis- } \\
\text { mos de analgesia y recompensa }\end{array}$ & $\begin{array}{l}\text { Gardner et al }(1998)^{39} \text { y } \\
\text { Hohmann et al }(2002)^{40}\end{array}$ \\
\hline & $\begin{array}{l}\text { Polimorfismo Pro129Thr del gen de la FAAH influye en } \\
\text { magnitud de analgesia placebo y en activación opioide } \\
\text { dada por EP }\end{array}$ & Pecina et al $(2015)^{42}$ \\
\hline Sistema serotoninérgico & $\begin{array}{l}\text { Polimorfismo G-703T TPH2 es predictor de EP en indi- } \\
\text { viduos con fobia social }\end{array}$ & Furmark et al $(2008)^{43}$ \\
\hline Sistema CCK & Proglumida potencia la analgesia generada por morfina & Lavigne et al (1989) \\
\hline & Se corrobora acción anti-opioidea de proglumida & Noble et al $2003^{35}$ \\
\hline \multirow[t]{7}{*}{ Sistema dopaminérgico } & $\begin{array}{l}\text { En Parkinson analgesia placebo genera actividad dopa- } \\
\text { minérgica en ganglios basales y NA }\end{array}$ & De la Fuente-Fernández et al $(2001)^{24}$ \\
\hline & $\begin{array}{l}\text { Interacción de sistema dopaminérgico mesilímbico con } \\
\text { SOE en analgesia placebo. Administración de placebo } \\
\text { genera activación dopaminérgica en terminales meso- } \\
\text { límbicas, núcleo caudado ventral, putámen ventral y NA }\end{array}$ & Scott et al $(2008)^{25}$ \\
\hline & $\begin{array}{l}\text { Polimorfismo rs } 4680 \text { del gen de la COMT genera me- } \\
\text { jores resultados en analgesia placebo en colon irritable } \\
\text { e individuos sanos }\end{array}$ & Hall et al $(2012)^{28}$ \\
\hline & $\begin{array}{l}\text { Polimorfismo rs6323 del gen de la MAO-A influye en } \\
\text { respuesta placebo de síntomas depresivos }\end{array}$ & Leuchter et al $(2009)^{30}$ \\
\hline & $\begin{array}{l}\text { Polimorfismo rs6280 presenta inferencia en mejoría } \\
\text { clínica en esquizofrénicos tratados con placebo }\end{array}$ & Bhathena et al $(2013)^{31}$ \\
\hline & $\begin{array}{l}\text { Polimorfismo rs1048261 del gen receptor de gluco- } \\
\text { corticoides y polimorfismo rs6609257 del gen MAO } \\
\text { generan mayor EP en individuos con depresión }\end{array}$ & Tiwari et al $(2013)^{32}$ \\
\hline & $\begin{array}{l}\text { Homocigotos para polimorfismo rs6265 del gen del } \\
\text { BDNF presentan mayor activación D2 y D3 mediada } \\
\text { por placebo }\end{array}$ & Pecina et al $(2015)^{33}$ \\
\hline
\end{tabular}

CCK: colecistoquinina, CCA: corteza cingulada anterior, CIA: corteza insular anterior, CPFDL: corteza prefrontal dorsolateral, LCR: líquido cefalorraquídeo EP: efecto placebo, NA: núcleo accumbens, MAO-A: monoamino oxidasa A, BDNF: factor neurotrófico derivado del cerebro, COMT: catecol-o-metiltransferasa, $\mathrm{RO}-\mu$ : receptor opioide $\mu$.

el fenómeno placebo incluyen áreas involucradas en la integración emocional y cognitiva (CCA), en la representación y modulación de los estados internos físicos y emocionales (CIA) y en la evaluación de la recompensa (NA) ${ }^{21}$. Posteriormente, un estudio de 2006 concluye que la experiencia emocional durante la percepción del dolor es un predictor importante para el desarrollo posterior del EP22.

Variaciones genotípicas del sistema opioide En el año 2014 se estudió el polimorfismo de nucleótido simple (SNP) A118G en el RO- $\mu$ (OPRM1) y su asociación con la respuesta psico- 
social, rasgos de personalidad y neurotransmisión opioide/dopaminérgica en el contexto de dolor y analgesia placebo. En esta investigación se halló que, al aplicar un estímulo doloroso, los portadores del alelo OPRM1 G -comparado con los homocigotos AA- mostraban una reducción de la disponibilidad de $\mathrm{RO}-\mu$ en regiones implicadas en la regulación del afecto y del dolor. Asimismo, los homocigotos AA mostraron una respuesta dopaminérgica reducida en el NA. Luego de la administración de placebo, los portadores de la variante $G$ mostraron mayores alteraciones del ánimo y menor activación inducida por placebo de RO- $\mu$ en CIA, amígdala, NA, tálamo y tronco encefálico, así como menor activación dopaminérgica de receptores D2 y D3 en el NA ${ }^{23}$.

\section{Placebo y sistema dopaminérgico}

En 2001, estudiando pacientes con enfermedad de Parkinson, se observó que bajo la administración de placebo existía actividad dopaminérgica en los ganglios basales y en el NA, asociándose respectivamente a la mejoría en el control motor y a la expectativa de mejoría reportada por los sujetos $^{24}$. Este antecedente, junto a la hipótesis de que el EP representa una forma de procesamiento de la recompensa, motiva el estudio de Scott et al, en 2008, donde se examina la relación entre la liberación dopaminérgica del NA y el SOE en la analgesia placebo, demostrando la participación del sistema dopaminérgico mesolímbico en la analgesia placebo (interaccionando con el SOE), constituyéndose como la primera documentación del rol del NA como diferenciador entre respondedores placebo altos o bajos, observándose una diferencia significativa en la actividad opioide de esta región cerebral entre ambos grupos. La administración de placebo fue asociada a la activación de la neurotransmisión dopaminérgica en terminales mesolímbicas, núcleo caudado ventral, putamen ventral y NA. La magnitud de la activación dopaminérgica en el NA fue correlacionada positivamente con la expectativa individual de analgesia, la actualización de esta expectativa y la magnitud de la analgesia percibida. Un hallazgo destacable fue la identificación de la sustancia gris periacueductal como participante en la activación del SOE inducida por placebo, considerando el rol de esta estructura en la modulación de las señales ascendentes del dolor hacia regiones telencefálicas ${ }^{25}$.
Variedad genotípica en el sistema dopaminérgico

Se ha evidenciado que la presencia del SNP rs4680 del gen de la catecol-o-metiltransferasa determina la disminución de la actividad de esta enzima $^{26}$, determinando la forma homocigota del alelo menos activo menor cantidad de dopamina en la corteza prefrontal. Lo anterior concuerda con aquellas investigaciones que evidencian la relevancia de este SNP en $\mathrm{el} \mathrm{EP}^{27}$ al hallar que su presencia genera mejores resultados en EP en individuos con colon irritable e individuos sanos ${ }^{28}$. El gen de la monoamino oxidasa A (MAO A) tiene rol en la oxidación de la dopamina y en el metabolismo de la serotonina ${ }^{29}$. Aquellos individuos que tienen solo el alelo rs6323 del SNP ligado a este gen presentan $75 \%$ de reducción de la actividad de la $\mathrm{MAO}$, de modo que aquellos individuos con alta actividad de la MAO presentan mayor reducción de síntomas depresivos producto del placebo ${ }^{30}$. En sujetos esquizofrénicos homocigotos para el SNP rs6280-polimorfismo que incrementa la afinidad de la dopamina por el receptor dopaminérgico DRD3- presentan mejores resultados al ser tratados con placebo que aquellos individuos a los que se les administra un antagonista del receptor $\mathrm{DRD}^{31}$. En depresión se analizaron distintos SNPs en variados genes para ver su asociación a la respuesta al tratamiento con placebo y con bupropion, se halló una mayor respuesta placebo en aquellos individuos con el SNPs rs 1048261 del gen receptor de glucocorticoides y en aquellos individuos con el SNP rs6609257 del gen $\mathrm{MAO}^{32}$. Por otro lado, el gen del factor neurotrófico derivado del cerebro posee el SNP rs6265 que genera reducción de la secreción de este factor y se ha visto que aquellos individuos homocigotos para este SNP presentan mayor activación de receptores D2 y D3 mediada por placebo ${ }^{33}$.

\section{Placebo y colecistoquinina}

En 1989, se demuestra que la proglumida (antagonista de la CCK) potencia la magnitud de la analgesia otorgada por morfina en dolor post-operatorio $^{34}$. En 1995, se observa que el EP puede ser parcialmente abolido por naloxona y potenciado por proglumida, sin embargo, esta potenciación solo se dio en respondedores placebo, sugiriendo que la proglumida necesita la acción del SOE para ejercer su efecto, instituyéndose así la CCK como un modulador con rol inhibitorio en la respuesta de analgesia placebo ${ }^{35}$. Posteriormente, se recono- 
ce a la CCK como un mediador en la hiperalgesia nocebo, al encontrar que la administración de proglumida es capaz de inhibir el dolor inducido en individuos previamente sugestionados con la capacidad algésica de una sustancia inerte ${ }^{36}$.

Considerando la capacidad ansiógena que poseen las expectativas negativas y el rol de la CCK en la modulación de la ansiedad ${ }^{37}$, los hallazgos previos sugieren que la acción de la proglumida se basa, al menos en parte, en los circuitos de modulación de ansiedad dependientes de CCK. Estos circuitos han sido localizados en zonas cerebrales como la amígdala, mientras que la acción pronociceptiva y antiopioide directa de la CCK ha sido localizada en áreas del tronco cerebral como la zona ventromedial del bulbo raquídeo ${ }^{38}$.

\section{Placebo y sistema cannabinoide}

El sistema cannabinoide, compuesto por los receptores $\mathrm{CB} 1$ and $\mathrm{CB} 2$ y sus ligandos endógenos, ha sido propuesto como participante en los mecanismos de recompensa ${ }^{36}$ y analgesia ${ }^{37}$. Los receptores $\mathrm{CB} 1$ y los $\mathrm{RO}-\mu$ están localizados en estructuras cerebrales implicadas en el control nociceptivo e interaccionan funcionalmente de modo sinérgico ${ }^{38}$. Considerando esta interacción, se realizó un estudio para comprender la relación entre ambos sistemas investigando la variante Pro129Thr del gen que codifica la amida hidrolasa de ácidos grasos (FAAH), enzima con rol protagónico en la degradación de canabinoides endógenos en la respuesta a analgesia placebo. Se encontró que aquellos homocigotos Pro129/Pro129 poseían mayor analgesia placebo y un estado anímico más positivo luego de la administración de placebo, así como mayor neurotransmisión opioide inducida por placebo ${ }^{39}$.

\section{Placebo y sistema serotoninérgico}

La participación de la serotonina como neurotransmisor colaborador en el EP surge de un estudio donde se pretendió examinar la variabilidad genética relacionada con la expresión de serotonina como predictor de respuesta placebo en individuos con fobia social ${ }^{40}$. Los genotipos de los pacientes fueron clasificados según la región polimórfica asociada al transportador de serotonina (5-HTTLPR) y el polimorfismo G-703T en el gen promotor de la triptófano hidroxilasa. Se encontró que la respuesta placebo estaba acompañada de una reducción en la actividad de la amígdala, sin embargo, esta menor actividad se daba sólo en sujetos que eran homocigotos para el alelo largo del 5-HTTLPR o la variante $\mathrm{G}$ del polimorfismo G-703TTPH2 el cual se presentó como predictor de respuesta placebo.

\section{Conclusiones}

La multiplicidad de hallazgos descritos en esta revisión hace evidente la necesidad de considerar las implicancias de estos en varios niveles:

- Al considerar la neurobiología del EP/EN es clara la implicancia de los sistemas opioide, dopaminérgico, colecistoquininérgico, canabinoide y serotoninérgico, comprometiendo a su vez estructuras de la corteza frontal (CPFDL y CCA, principalmente) y sistema límbico. Estas estructuras y sistemas de neurotransmisión involucrados se correlacionan con procesos cognitivos y emocionales relevantes para la ocurrencia de ambos procesos, siendo la identificación sistemática de estas estructuras y sistemas un camino para erradicar el halo de misterio de ambos fenómenos, acercándolos al escrutinio científico. La reciente identificación de polimorfismos que impactan las vías opioide, dopaminérgica y serotoninérgica -modificando la potencial respuesta individual al placebo- constituye una interesante corriente de investigación, siendo el entendimiento en este campo una promesa de generar nuevas posibilidades de maximizar resultados de tratamientos en ciertas enfermedades y nuevas oportunidades de evaluar con mayor validez la real eficacia de agentes farmacológicos.

- Al reunir el conocimiento del EP/EN en la práctica clínica, es posible apreciar la magnitud de ambos fenómenos abarcando desde cuadros quirúrgicos a psiquiátricos, desde el temblor al dolor. A la luz de estos hallazgos, debiéramos considerar de rutina la presencia del EP/EN en el diario quehacer del clínico; actualmente el paciente es un sujeto cada vez con más información, llevando en su interior diversas aprehensiones respecto de los tratamientos disponibles, siendo la consideración de estas aprehensiones de vital importancia al evaluar aquellos resultados terapéuticos donde el EP ha mostrado importancia: dolor, 
angustia, temblor. En este sentido, el cómo se comunica una indicación, esto es, la claridad y seguridad con que esta indicación se realiza, podrá tener impacto en los resultados de esta. Siendo consecuentes con lo anterior, al momento de evaluar los resultados de cada intervención es necesario no perder de vista el EP/EN tanto en los efectos positivos como negativos. Muchos pacientes refieren efectos colaterales con prácticamente toda indicación, en cuyo caso debemos revisar las creencias y expectativas que estas personas tienen en relación al tratamiento. Por otra parte, cuando la temporalidad y estabilidad de los resultados no se correlacionan adecuadamente con los efectos terapéuticos esperados, se debe revisar el rol del EP/EN. Este análisis puede permitir tomar mejores decisiones en el manejo futuro del caso.

- La magnitud y relevancia del EP/EN debiera llevarnos a desarrollar líneas de investigación clínica para su mejor comprensión y aplicación justamente en este ámbito, en que nos encontramos con el objeto de nuestro esfuerzo, los pacientes.

Finalmente, es relevante llamar la atención sobre la importancia de transmitir a los estudiantes de Medicina los conocimientos que les permitan identificar el EP/EN, de manera de utilizarlos como herramienta a favor del proceso terapéutico.

\section{Referencias}

1. Kradin R. Placebo Response: A Consideration of its Role in Therapeutics. Curr Psychiatry Rep 2011; 13 (1): 3742.

2. Rosenzweig P, Brohier S, Zipfel A. The placebo effect in healthy volunteers: influence of experimental conditions on the adverse events profile during phase I studies. Clin Pharmacol Ther 1993; 54 (5): 578-83.

3. Hróbjartsson A, Gøtzsche PC. Placebo interventions for all clinical conditions. Cochrane Database Syst Rev 2010 (1)

4. Häuser W, Bartram-Wunn E, Bartram C, Reinecke H, Tölle T. Systematic review: placebo response in drug trials of fibromyalgia syndrome and painful peripheral diabetic neuropathy, magnitude and patient-related predictors. Pain 2011; 152 (8): 1709-17.

5. Khan A, Kolts RL, Rapaport MH, Krishnan KR, Brod- head AE, Browns WA. Magnitude of placebo response and drug-placebo differences across psychiatric disorders. Psychol Med 2005; 35 (5): 743-9.

6. Rief W, Nestoriuc Y, Weiss S, Welzel E, Barsky AJ, Hofmann SG. Meta-analysis of the placebo response in antidepressant trials. Journal of Affective Disorders 2009; 118 (1-3): 1-8.

7. Moseley JB, O’Malley K, Petersen NJ, Menke TJ, Brody $\mathrm{BA}$, Kuykendall DH, et al. A controlled trial of arthroscopic surgery for osteoarthritis of the knee. N Engl J Med 2002; 347: 81-8.

8. Geers AL, Helfer SG, Kosbab K, Weiland PE, Landry SJ. Reconsidering the role of personality in placebo effects: dispositional optimism, situational expectations, and the placebo response. J Psychosom Res 2005; 58 (2): 121-7.

9. Zubieta JK, Stohler CS. Neurobiological Mechanisms of Placebo Responses. Ann N Y Acad Sci 2009; 1156: 198-210.

10. Atlas LY, Wager TD. A meta-analysis of brain mechanisms of placebo analgesia: consistent findings and unanswered questions. Handb Exp Pharmacol 2014; 225: 37-69.

11. Wager TD, Rilling JK, Smith EE, Sokolik A, Casey KL, Davidson RJ, et al. Placebo-induced changes in FMRI in the anticipation and experience of pain. Science 2004; 303: 1162-7.

12. Krummenacher P, Candia V, Folkers G, Schedlowski M, Schönbächler G. Prefrontal cortex modulates placebo analgesia. Pain 2010; 148 (3): 368-74.

13. Benedetti F, Arduino C, Costa S, Vighetti S, Tarenzi L, Rainero I, et al. Loss of expectation-related mechanisms in Alzheimer's disease makes analgesic therapies less effective. Pain 2006; 121 (1-2): 133-44.

14. Lorenz J, Hauck M, Paur RC, Nakamura Y, Zimmermann R, Bromm B, et al. Cortical correlates of false expectations during pain intensity judgments-a possible manifestation of placebo/nocebo cognitions. Brain Behav Immun 2005; 19 (4): 283-95.

15. Bingel U, Wanigasekera V, Wiech K, Ni Mhuircheartaigh R, Lee MC, Ploner M, et al. The effect of treatment expectation on drug efficacy: imaging the analgesic benefit of the opioid remifentanil. Sci Transl Med 2011; 3 (70): 70ra14.

16. Levine JD, Gordon NC, Fields HL. The mechanisms of placebo analgesia. The Lancet 1978; 2: 654-7.

17. Levine JD, Gordon NC. Influence of the method of drug administration on analgesic response. Nature 1984; 312 (5996): 755-6.

18. Amanzio M, Benedetti F. Neuropharmacological dissection of placebo analgesia: expectation-activated opioid 
systems versus conditioning-activated specific sub-systems. J Neurosci 1999; 19 (1): 484-94.

19. Lipman JJ, Miller BE, Mays KS, Miller MN, North WC, Byrne WL. Peak B endorphin concentration in cerebrospinal fluid: reduced in chronic pain patients and increased during the placebo response. Psychopharmacology (Berl). 1990; 102 (1): 112-6.

20. Petrovic $P$, Ingvar M. Imaging cognitive modulation of pain processing. Pain 2002; 95 (1-2): 1-5.

21. Zubieta JK, Bueller JA, Jackson LR, Scott DJ, Xu Y, Koeppe RA, et al. Placebo effects mediated by endogenous opioid activity on mu-opioid receptors. J Neurosci. 2005; 25 (34): 7754-62.

22. Zubieta JK, Yau WY, Scott DJ, Stohler CS. Belief or need? Accounting for individual variations in the neurochemistry of the placebo effect. Brain Behav Immun 2006; 20 (1): 15-26.

23. Peciña M, Love T, Stohler CS, Goldman D, Zubieta JK. Effects of the Mu opioid receptor polymorphism (OPRM1 A118G) on pain regulation, placebo effects and associated personality trait measures. Neuropsychopharmacology 2015; 40 (4): 957-65.

24. De la Fuente-Fernández R, Ruth TJ, Sossi V, Schulzer M, Calne DB, Stoessl AJ. Expectation and dopamine release: Mechanism of the placebo effect in Parkinson's disease. Science 2001; 293 (5532): 1164-6.

25. Scott DJ, Stohler CS, Egnatuk CM, Wang H, Koeppe RA, Zubieta JK. Placebo and nocebo effects are defined by opposite opioid and dopaminergic responses. Arch Gen Psychiatry 2008; 65 (2): 220-31.

26. Lotta T, Vidgren J, Tilgmann C, Ulmanen I, Melén K, Julkunen I, et al. Kinetics of human soluble and membrane bound catechol O-methyltransferase: a revised mechanism and description of the thermoabile variant of the enzime. Biochemistry 1995; 34 (13), pp 4202-10.

27. Tammimaki A, Mannisto PT. Catechol-O-methyltrasnferase gene polymorphism and chronic human pain: a sistematic review and meta-analysis. Pharmacogenet Genomics 2012; 22 (9): 673-91.

28. Hall KT, Lembo AJ, Kirsch I, Ziogas DC, Douaiher $\mathrm{J}$, Jensen $\mathrm{KB}$, et al. Catechol-O-methyltransferase val158met polymorphism predicts placebo effect in irritable bowel syndrome. 2012. PLoS One 2012; 7 (10): e48135.

29. Mickey BJ, Ducci F, Hodgkinson CA, Langenecker SA, Goldman D, Zubieta JK. Monoamine oxidase A genotype predicts human serotonin $1 \mathrm{~A}$ receptor availability in vivo. J Neurosci 2008; 28 (44): 11354-9.

30. Leuchter AF, McCracken JT, Hunter AM, Cook IA, Alpert JE. Monoamine oxidase a and catechol-o-methyltransferase functional polymorphisms and the placebo response in major depressive disorder. J Clin Psychopharmacol 2009; 29 (4): 372-7.

31. Bhathena A, Wang Y, Kraft JB, Idler KB, Abel SJ, Holley-Shanks RR, et al. Association of dopamine-related genetic loci to dopamine D3 receptor antagonist ABT925 clinical response. Transl Psychiatry 2013; 3: e245.

32. Tiwari AK, Zai CC, Sajeev G, Arenovich T, Müller DJ, Kennedy JL. Analysis of 34 candidate genes in bupropion and placebo remission. Int J Neuropsychopharmacol 2013; 16 (4).

33. Peciña M, Martínez-Jauand M, Love $T$, Heffernan J, Montoya P, Hodgkinson C. Valence-specific effects of BDNF Val66Met polymorphism on dopaminergic stress and reward processing in humans. J Neurosci 2014; 34 (17): 5874-81.

34. Lavigne GJ, Hargreaves KM, Schmidt EA, Dionne RA. Proglumide potentiates morphine analgesia for acute postsurgical pain. Clin Pharmacol Ther 1989; 45 (6): 666-73.

35. Noble F, Roques BP. The role of CCK2 receptors in the homeostasis of the opioid system. Drugs Today (Barc) 2003; 39 (11): 897-908.

36. Benedetti F, Amanzio M, Casadio C, Oliaro A, Maggi G. Blockade of nocebo hyperalgesia by the cholecystokinin antagonist proglumide. Pain 1997; 71 (2): 135-40.

37. Rotzinger S, Vaccarino FJ. Cholecystokinin receptor subtypes: role in the modulation of anxiety-related and reward-related behaviours in animal models. J Psychiatry Neurosci 2003; 28 (3): 171-81.

38. Benedetti F, Lanotte M, Lopiano L, Colloca L. When words are painful: unraveling the mechanisms of the nocebo effect. Neuroscience 2007; 147 (2): 260-71.

39. Gardner EL, Vorel SR. Cannabinoid transmission and reward-related events. Neurobiology of disease 1998; 5 (6 Pt B): 502-33.

40. Hohmann AG. Spinal and peripheral mechanisms of cannabinoid antinociception: behavioral, neurophysiological and neuroanatomical perspectives. Chemistry and physics of lipids 2002; 121 (1-2): 173-90.

41. Salio C, Fischer J, Franzoni MF, Mackie K, Kaneko T, Conrath M. CB1-cannabinoid and mu-opioid receptor co-localization on postsynaptic target in the rat dorsal horn. Neuroreport 2001; 12 (17): 3689-92.

42. Peciña M, Martínez-Jauand M, Hodgkinson C, Stohler CS, Goldman D, Zubieta JK. FAAH selectively influences placebo effects. Mol Psychiatry 2014; 19 (3): 385-91.

43. Furmark T, Appel L, Henningsson S, Åhs F, Faria V, Linnman C, et al. A link between serotonin-related gene polymorphisms, amygdala activity, and placebo-induced relief from social anxiety. J Neurosci 2008; 28 (49): 13066-74. 